\title{
DSP-BASED IMPLEMENTATION OF A REAL-TIME DOA ESTIMATOR FOR UNDERWATER ACOUSTIC SOURCES
}

\author{
Sheng-Yun Hou \\ Department of Electrical Engineering, National Taiwan Ocean University, Keelung, Taiwan, R.O.C. Department of \\ Electronic Engineering, Hwa Hsia Institute of Technology, Chung Ho, Taipei, Taiwan, R.O.C. \\ Shun-Hsyung Chang \\ Department of Microelectronic Engineering, National Kaohsiung Marine University, Kaohsiung, Taiwan, R.O.C. \\ Hsien-Sen Hung \\ Department of Electrical Engineering, National Taiwan Ocean University, Keelung, Taiwan, R.O.C., \\ b0221@mail.ntou.edu.tw \\ Jiang-Yao Chen \\ Department of Electrical Engineering, National Taiwan Ocean University, Keelung, Taiwan, R.O.C.
}

Follow this and additional works at: https://jmstt.ntou.edu.tw/journal

Part of the Electrical and Computer Engineering Commons

\section{Recommended Citation}

Hou, Sheng-Yun; Chang, Shun-Hsyung; Hung, Hsien-Sen; and Chen, Jiang-Yao (2009) "DSP-BASED IMPLEMENTATION OF A REAL-TIME DOA ESTIMATOR FOR UNDERWATER ACOUSTIC SOURCES," Journal of Marine Science and Technology: Vol. 17: Iss. 4, Article 10.

DOI: 10.51400/2709-6998.1989

Available at: https://jmstt.ntou.edu.tw/journal/vol17/iss4/10

This Research Article is brought to you for free and open access by Journal of Marine Science and Technology. It has been accepted for inclusion in Journal of Marine Science and Technology by an authorized editor of Journal of Marine Science and Technology. 


\section{DSP-BASED IMPLEMENTATION OF A REAL-TIME DOA ESTIMATOR FOR UNDERWATER ACOUSTIC SOURCES}

\section{Acknowledgements}

This work was supported by National Science Council of TAIWAN under contracts NSC 96-2221-E-022-016- and NSC 95-2221-E-022-017-. The authors would like to thank unknown reviewers with invaluable comments and suggestions. Thanks also go to Mr. C. H. Liu, Mr. M. C. Chuang, and Mr. Y. D. Huang for their efforts in setting up the experimental equipments during the sea trial experiments. 


\title{
DSP-BASED IMPLEMENTATION OF A REAL-TIME DOA ESTIMATOR FOR UNDERWATER ACOUSTIC SOURCES
}

\author{
Sheng-Yun Hou****, Shun-Hsyung Chang***, Hsien-Sen Hung*, and Jiang-Yao Chen*
}

\begin{abstract}
Key words: digital signal processor (DSP), direction-of-arrival estimation, array signal processing, underwater acoustic channel.
\end{abstract}

\begin{abstract}
Many direction-of-arrival (DOA) estimation algorithms for acoustic sources based on sensor array measurements have been well developed and verified via computer simulations and/or small-scale water trough experiments. However, these results are not in practical use because the testing scenarios may not reflect real underwater environments. In order to have a better understanding of DOA estimation in sea experiments, a prototype of DSP-based acoustic DOA estimator is designed and implemented. The prototype system is based on TMS320VC33 digital signal processor and is validated by both computer simulation and experiments performed on the shallow water of Bi-Sha fishery harbor located near the coastal region of Keelung, Taiwan.
\end{abstract}

\section{INTRODUCTION}

Direction-of-arrival (DOA) estimation is widely used for target localization and tracking in many areas such as wireless communications, radar and sonar [3]. During the past twenty years, various DOA estimation methods based on the measurements of sensor array were attempted to achieve higher resolution capability while reducing computational complexity. Most papers in the literature deal with the theoretical development for DOA estimation and its verification via computer simulation [2, 4-7]. Very few describe the actual implementation of DOA estimation system and its use in practical real-time sea trial experiments. Thanks to the rapid

Paper submitted 05/02/08; revised 09/03/08; accepted 12/13/08. Author for correspondence: Hsien-Sen Hung (e-mail: b0221@mail.ntou.edu.tw).

*Department of Electrical Engineering, National Taiwan Ocean University, Keelung, Taiwan, R.O.C.

**Department of Electronic Engineering, Hwa Hsia Institute of Technology, Chung Ho, Taipei, Taiwan, R.O.C.

***Department of Microelectronic Engineering, National Kaohsiung Marine University, Kaohsiung, Taiwan, R.O.C. progress of VLSI and computer technologies, implementation of the aforementioned system based on digital signal processor (DSP) has become possible nowadays. In this paper, we design and implement a real-time DOA estimation system using TMS320VC33 processor chip as a core processor, AD7891 chip as analog-to-digital converter, and the associated hardware circuits. The prototype system is then tested via computer simulation and experiments conducted in the shallow water of Bi-Sha fishery harbor located near the coastal region of Keelung, Taiwan.

\section{HARDWARE DESIGN}

The block diagram of the developed prototype system, is depicted in Fig. 1. The TMS320VC33 processor, produced by Texas Instruments (TI), plays a central role for data retrieval and program execution $[8,9]$. The nine-channel analog-todigital converter, utilizing the AD7891 chip (produced by Analog Device [10]), is to transform analog acoustic signals into digital codes. In the actual system (not shown in Fig. 1.), four 8104 receive hydrophones, produced by B\&K, are used and connected to the LF398 sample-and-hold IC for simultaneously sampling the received analog signals [11]. For data/program storage and retrieval, the IDT-71V242-10 SRAM and AT29LV040A-150 Flash memory, produced by IDT and ATMEL respectively, are adopted $[12,13]$. In addition, CPLD is incorporated for convenient and flexible design of decoder device. The completed circuit board for real-time DOA estimator is shown in Fig. 2.

\section{SOFTWARE DESIGN}

The TMS320VC33 processor is a 32-bit floating point DSP chip, which allows users to utilize both $\mathrm{C}$ and assembly programming languages for program development [14]. Firstly, the original program (DOA estimation algorithm) written in C or assembly language is coded by an editor. Then it is compiled/assembled and linked to produce an executable COFF file, a common object file format with .out as an extension filename, which can be loaded onto (software) simulator XDS510 or an (hardware) emulator for error debugging. After algorithm validation, the .out file is further transformed via 


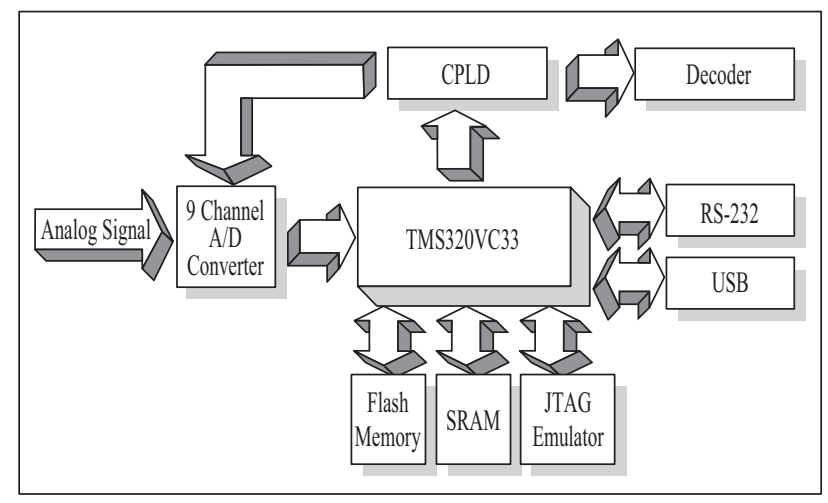

Fig. 1. Block diagram of real-time DOA estimator VC33 prototype system.

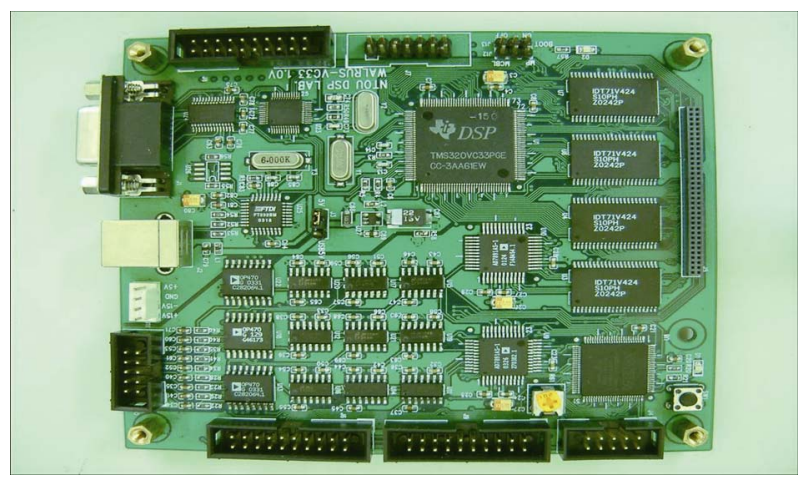

Fig. 2. Circuit board of real-time DOA estimator VC33 prototype system.

HEX30.EXE into .HEX file, accessible by EPROM. Finally, the .HEX file is loaded into the EPROM of the target hardware with an IC writer to complete the DSP algorithm development cycle.

In the following content, a data model that is adopted in the DOA estimation algorithms is presented, and then followed by a brief review on each DOA estimation algorithm used for the purpose of performance evaluation.

Consider that there are $M$ acoustic sources from which narrowband signals are emitted, propagated through an underwater acoustic channel and received by a receive array of $L$ $(>M$ ) hydrophones (sensors). At time $t$, the output of the sensor array is given by

$$
\mathbf{r}(t)=\mathbf{A s}(t)+\mathbf{n}(t)
$$

where $\mathbf{r}(t)=\left[r_{1}(t), \ldots, r_{L}(t)\right]^{T}, \mathbf{s}(t)=\left[s_{1}(t), \ldots, s_{M}(t)\right]^{T}$, and $\mathbf{n}(t)=$ $\left[n_{1}(t), \ldots, n_{L}(t)\right]^{T}$ are the output data, source signal, and ambient noise vectors, respectively. The superscript $T$ denotes the transpose operator. The $L \times M$ direction matrix $\mathbf{A}=\left[\mathbf{a}_{1}, \ldots\right.$, $\mathbf{a}_{M}$ ] has the column vectors in the form of

$$
\mathbf{a}_{m}=\left[1, e^{-j \frac{2 \pi}{\lambda} d \sin \theta_{m}}, \ldots, e^{-j \frac{2 \pi}{\lambda}(L-1) d \sin \theta_{m}}\right]^{T}, \quad m=1, \ldots, M
$$

where $\mathbf{a}_{m}$ denotes the direction vector of the $m$ th source with incident angle $\theta_{m}$, provided that a uniform linear array (ULA) with interelement spacing $d$ is used, $\lambda$ is the wavelength, and all sources located in the far field are coplanar with the array. Assuming that $N$ snapshots of array output data $\mathbf{r}(t), t=1, \ldots$, $N$, are available, the correlation matrix $\mathbf{R}=E\left[\mathbf{r r}^{H}\right]$ can be estimated by the sample correlation matrix.

$$
\hat{\mathbf{R}}=\frac{1}{N} \sum_{t=1}^{N} \mathbf{r}(t) \mathbf{r}^{H}(t)
$$

The superscript $H$ denotes the Hermitian transpose.

\section{MUSIC Algorithm [6]}

The MUSIC algorithm is one of the subspace-based methods, which has high resolution property for DOA estimation of multiple sources. MUSIC is known to yield asymptotically unbiased DOA estimate, when the sources are uncorrelated or partially correlated. However, when the signals are perfectly correlated (coherent), MUSIC encounters significant performance degradation [7]. The DOAs are determined as the local peak locations of the pseudo-spectrum

$$
S(\theta)=\frac{1}{\mathbf{a}^{H}(\theta) \mathbf{E}_{N} \mathbf{E}_{N}^{H} \mathbf{a}(\theta)}
$$

where $\mathbf{a}(\theta)=\left[1, e^{-j \frac{2 \pi}{\lambda} d \sin \theta}, \ldots, e^{-j \frac{2 \pi}{\lambda}(L-1) d \sin \theta}\right]^{T}$ is the array manifold with $\theta \in\left[-90^{\circ}, 90^{\circ}\right], \mathbf{E}_{N}$ is the noise subspace spanned by the noise eigenvectors associated with the $L-M$ smallest eigenvalues of $\mathbf{R}$. In practice, the noise eigenvectors are chosen from the eigen-structure of the sample correlation matrix $\hat{\mathbf{R}}$. Extensive search on the pseudo-spectrum over $\theta \in\left[-90^{\circ}, 90^{\circ}\right]$ is needed for finding local peaks.

\section{Reduced-Order Root-MUSIC (RORM) [4]}

Root-MUSIC, a variant of MUSIC, is a polynomial rooting algorithm for high resolution direction finding [1]. For ULA, the null spectrum of (4) in $\mathrm{z}$-domain can be defined as

$$
D(z)=\mathbf{a}^{H}\left(z^{-1}\right) \mathbf{E}_{N} \mathbf{E}_{N}^{H} \mathbf{a}\left(z^{*}\right)
$$

where the superscript $*$ denotes the complex conjugation, $\mathbf{a}(z)=$ $\left[1 z^{-1} \ldots z^{-(L-1)}\right]^{H}$ is the array manifold in $z$-domain. The zeros of $D(z)$ have a conjugate symmetry property. In practice, $D(z)$ is replaced with its estimate $\hat{D}(z)$ obtained by the eigen-decomposition of $\hat{\mathbf{R}}$. Firstly, Root-MUSIC finds $L$ zeros, located inside or on the unit circle, of $\hat{D}(z)$ that are closest to the unit circle. Finally, the DOAs are determined from the phase angles of these selected zeros.

Root-MUSIC is computationally more efficient than MUSIC since the DOAs can be directly read off from the arguments 
of the signal-related roots of a polynomial. Nevertheless, Root-MUSIC requires finding roots of a polynomial with degree $2(L-1)$. In order to further reduce computational complexity, the reduced order Root-MUSIC (RORM) algorithm is proposed [4], which only needs to find roots of a polynomial of order $2 M$. It requires finding all the roots of a polynomial with an order equaling twice the number of propagating signals. The removal of noise-related roots is achieved by QR factorization and Gauss elimination on $\mathbf{E}_{N}$. These roots are all signal-related, thereby providing a significant reduction of computations, particularly when the number of propagating signals is much less than the number of sensors.

The RORM algorithm can be outlined as follows.

- Perform QR factorization on $\mathbf{E}_{N}^{H}$ to have a lower triangular matrix $\mathbf{L}$,

- Obtain $\mathbf{U}_{N}$ in the diagonal-stripe form by using Gauss elimination on the column vectors of $\mathbf{L}$,

- Form a $2 M$-degree polynomial

$$
C(z)=\mathbf{a}^{H}\left(z^{-1}\right) \mathbf{U}_{N} \mathbf{T}(z) \mathbf{T}^{H}\left(z^{-1^{*}}\right) \mathbf{U}_{N}^{H} \mathbf{a}\left(z^{*}\right)
$$

where $\mathbf{T}(z)=\operatorname{diag}\left\{1, z^{-1}, \ldots, z^{-(L-M)+1}\right\}$ is a diagonal matrix, - Find $M$ zeros of $C(z)$ which are inside or on the unit circle,

- Obtain DOA estimates from the phase angles of these calculated zeros.

\section{Spatial Smoothing MUSIC (SSM) [7]}

MUSIC and RORM are inherently limited to the case that uncorrelated or partially correlated signals are received. In practical situations, received signals are often highly correlated or even coherent due to multiple reflections. For the case that all signals are coherent, the spatially smoothing MUSIC (SSM) method is proposed [7]. Its solution is based on a preprocessing scheme referred to as spatial smoothing that essentially decorrelates the signal coherence before MUSIC is invoked.

Let a ULA with $L$ sensors $\{1, \ldots, L\}$ be divided into overlapping subarrays of size $p$, with sensors $\{1, \ldots, p\}$ forming the first subarray, sensors $\{2, \ldots, p+1\}$ forming the second subarray, etc. Suppose the correlation matrix of the $j$ th subarray, $j=1, \ldots, U(=L-p+1)$, is denoted as $\mathbf{R}_{j}$. Then the spatially smoothed correlation matrix $\overline{\mathbf{R}}$ is obtained by averaging over $U$ correlation matrices associated with these overlapping subarrays, i.e., $\overline{\mathbf{R}}=\frac{1}{U} \sum_{j=1}^{U} \mathbf{R}_{j}$.

\section{Least-Squares (LS) [5]}

The method of [5] is called least squares (LS) in this paper because it involves a solution based on LS criterion. The LS method provides an alternative means to tackle the signal coherence problem mentioned above.

Consider the noise-free correlation matrix $\mathbf{R}_{y}$, which is $\mathbf{R}_{y}=$
$\mathbf{A} \mathbf{R}_{s} \mathbf{A}^{H}$ with the signal correlation matrix $\mathbf{R}_{s}=E\left[\mathbf{s s}^{H}\right]$. Let the $(i, j)$ element of $\mathbf{R}_{y}$ be denoted as $\gamma_{i j}$. The LS algorithm is outlined as follows.

- Estimate $\mathbf{R}_{y}$ from the sample correlation matrix $\hat{\mathbf{R}}$ of the sensor array output.

- Define the following matrices and vectors

$$
\begin{gathered}
\mathbf{Q}_{(i)}=\left[\begin{array}{cccc}
\gamma_{i, 1} & \gamma_{i, 2} & \cdots & \gamma_{i, M} \\
\gamma_{i, 2} & \gamma_{i, 3} & \cdots & \gamma_{i, M+1} \\
\vdots & \vdots & \ddots & \vdots \\
\gamma_{i, L-M} & \gamma_{i, L-M+1} & \cdots & \gamma_{i, L-1}
\end{array}\right] \\
\mathbf{Q}=\left[\mathbf{Q}_{(1)}, \mathbf{Q}_{(2)}, \cdots, \mathbf{Q}_{(L)}\right]^{T} \\
\mathbf{c}_{(i)}=\left[\begin{array}{c}
\gamma_{i, M+1} \\
\gamma_{i, M+2} \\
\vdots \\
\gamma_{i, L}
\end{array}\right] \\
\mathbf{c}=\left[\mathbf{c}_{(1)}, \mathbf{c}_{(2)}, \cdots, \mathbf{c}_{(L)}\right]^{T}
\end{gathered}
$$

And calculate the vector $\hat{\mathbf{Y}}_{M}$ as the LS solution to $\mathbf{Q Y} \mathbf{Y}_{M}=\mathbf{c}$, namely

$$
\hat{\mathbf{Y}}_{M}=\left(\mathbf{Q}^{H} \mathbf{Q}\right)^{-1} \mathbf{Q}^{H} \mathbf{c}
$$

- Estimate the DOAs by locating the peaks of the spatial spectrum

$$
P(\theta)=\frac{1}{\left|\mathbf{W}^{T}(\theta) \hat{\mathbf{Y}}\right|^{2}}
$$

where $\mathbf{W}(\theta)=\left[1, w, \ldots, w^{M}\right]^{T}, \quad w=\exp \left(j \frac{2 \pi}{\lambda} d \sin \theta\right)$, and $\hat{\mathbf{Y}}=\left[-\hat{\mathbf{Y}}_{M}^{*} ; 1\right]$. Extensive search on the pseudo-spectrum over $\theta \in\left[-90^{\circ}, 90^{\circ}\right]$ is needed for finding local peaks.

The LS method can achieve simultaneous detection and DOA estimation by comparing over-modeled pseudo-spectra for coherent sources. It is known to be robust against overmodeling. The minimum number of sensor required is twice the number of coherent signals.

\section{SYSTEM VERIFICATION}

\section{Verification of DOA Estimation Algorithms}

For sea trial experiments, four B\&K 8104 hydrophones are formed as an uniform linear array (ULA), as shown in Fig. 3. The ULA with the interelement spacing of half wavelength 


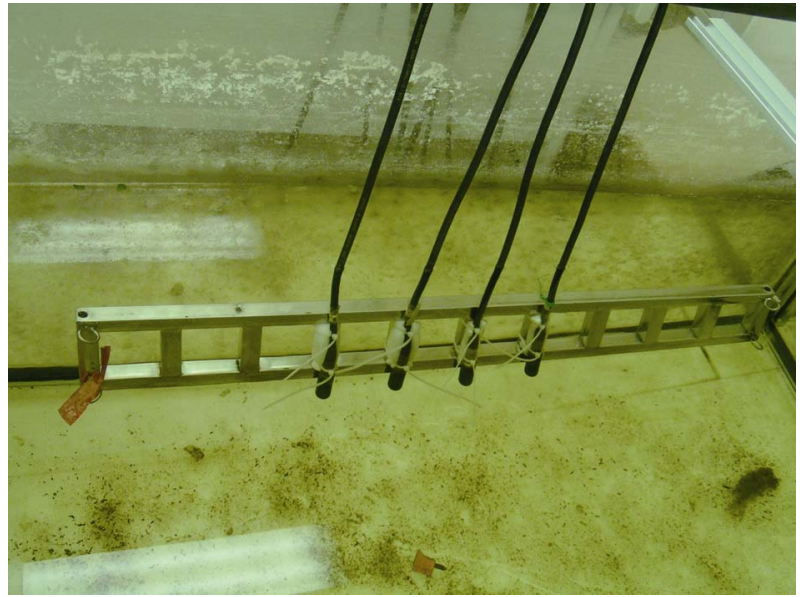

Fig. 3. Uniform linear array.

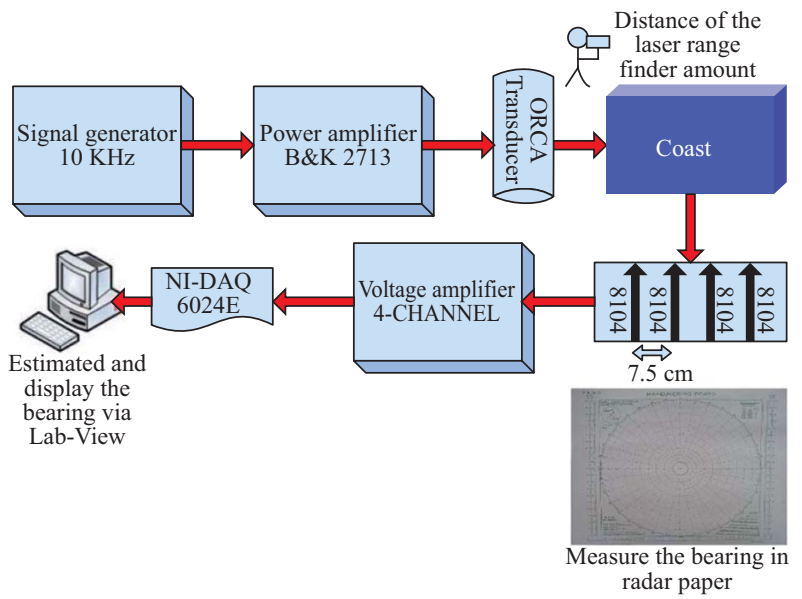

Fig. 4. Sea-trial experimental flow chart for Lab-View system.

$(7.5 \mathrm{~cm})$ is deployed to receive acoustic signals propagated in the sea near the coastal region of Bi-Sha Fishery harbor.

As shown in Fig. 4, the received array signal is passed through a 4-channel voltage amplifier, obtained by the data acquisition device NI-DAQ 6024E, and accessed and processed by the Lab-View system for DOA estimation. In addition, an omnidirectional transducer, produced by ORCA, is used as an acoustic sound projector. Prior to probing acoustic waves in the sea, it is connected to a power amplifier for power amplification of $20 \mathrm{~dB}$ as well as a signal generator for output of a sinusoidal signal with frequency of $10 \mathrm{KHz}$. The distance between the acoustic probe and the ULA is about 152 meters. All the hydrophones are submerged 2 meters in the sea. The signal-to-noise ratio is defined as $\mathbf{S N R}=10 \log \left(\mathbf{S} / \sigma^{2}\right)$, where $\mathbf{S}$ and $\sigma^{2}$ are the signal and noise powers measured at the output of the sensor array when a signal is and isn't in transmission respectively. Five hundred measurements (snapshots) are sampled at the sensor array output for DOA estimation.

The four DOA algorithms (MUSIC [6], Reduced-order Root MUSIC (RORM) [4], Spatial Smoothing MUSIC (SSM) [7],

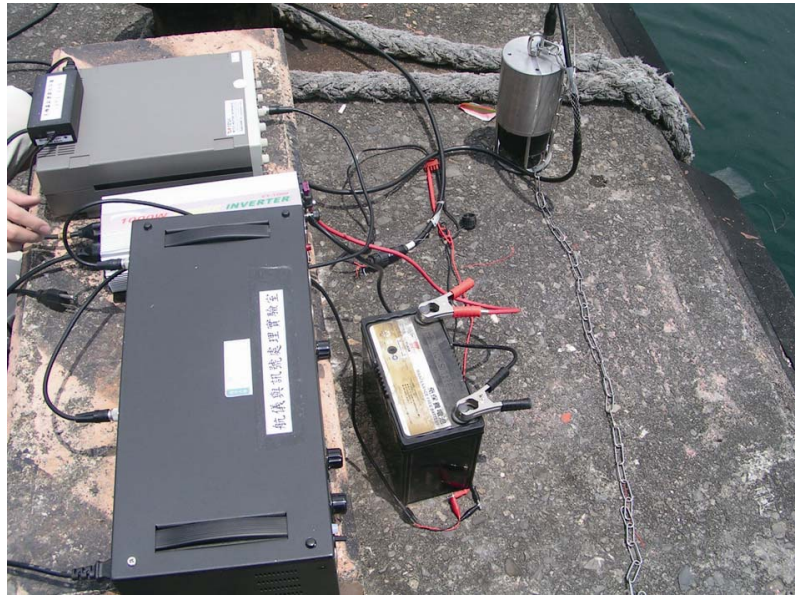

Fig. 5. Equipment setup at transmitter site.

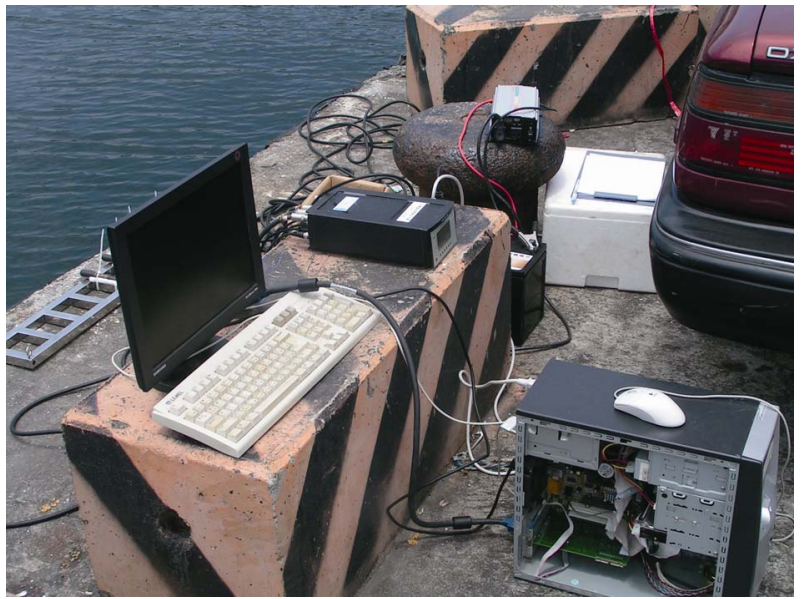

Fig. 6. Equipment setup at receiver site.

and Least-Squares (LS) [5]) are employed for performance comparison. Each algorithm is furnished to be executable by the Lab-View system and its associated hardware. Since the receive signals of the sensor array are in the form of real numbers, the Hilbert Transform is performed to transform the real signals into complex signals, which are required in each of the chosen DOA algorithms. Figures 5 and 6 show the photos of equipment setup at the transmitter and receiver sites, respectively.

As shown in Fig. 7, the OCAR transducer is assumed to be coplanar with the receive hydrophone array. The first hydrophone is chosen as a reference point, which corresponds to the center of concentric circles on the radar paper. The DOA of the sound projector is measured by the use of the ruler and trammel, and the range between the sound projector and the reference hydrophone is obtained with the aid of a laser range finder. The actual DOA and range of the sound projector in this experiment is 24.5 degrees and 152 meters, respectively, clearly indicated in the radar paper. As shown in Fig. 8, the estimated DOAs, obtained by the use of the four DOA algorithms 


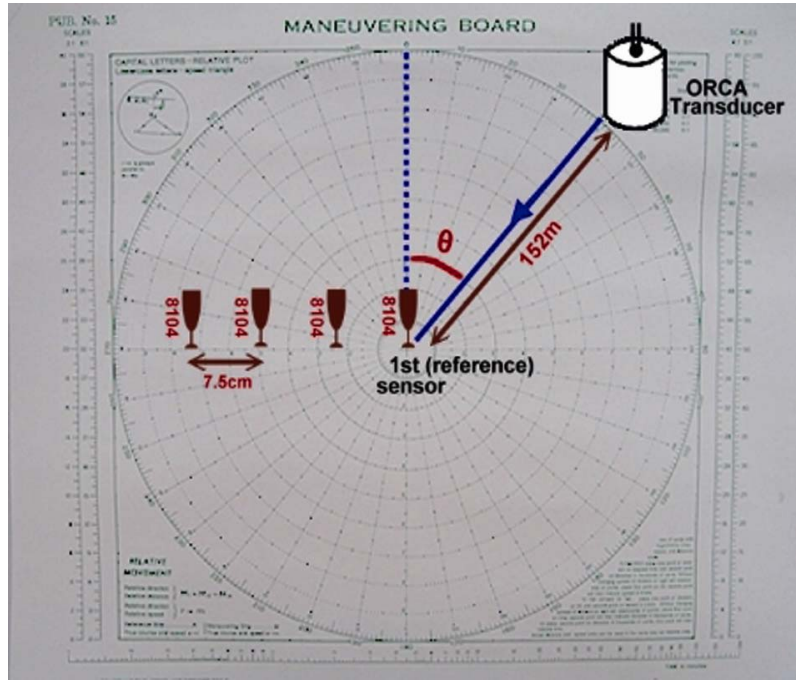

Fig. 7. DOA and range are recorded on the radar paper.

(MUSIC, RORM, SSM and LS), are 19.5, 19.5, 20.2, and 19.2 degrees respectively at $\mathrm{SNR}=4.4 \mathrm{~dB}$, using the system shown in Fig. 4. All of the four algorithms exhibit nearly the same performance while a bias of about 4 degrees exists. The bias may come from position errors of the transducers which are deployed in the shallow region near the seashore of the $\mathrm{Bi}$-Sha fishery harbor. Because turnaround of the fishing boats at the seaport often affects the turbulence of the water current, the random shifting of the deployed transducers appears leading to the position errors.

\section{Verification of the Prototype Real-Time DOA Estimator}

As shown in Fig. 9, our completed prototype real-time DOA estimator (abbreviated as VC33) is used to replace the LabView system and its associated DAQ hardware portrayed in Fig. 4. Only the LS algorithm is implemented and tested due to the following reasons. First, the LS algorithm can deal with coherent signals which may be caused by multiple paths of the underwater acoustic channel. Second, the LS algorithm is easier to be implemented in $\mathrm{C}$ codes, because it does not require complicated operations such as eigen-decomposition or QR factorization. Figure 10 shows the pseudo-spectra obtained from Lab-View system (upper half) and VC33 prototype (lower half) using the TI's Code Composer Studio (CCS). The location where the peak occurs determines the estimated DOA. The VC33 prototype gives the estimated DOA of 18 degrees whereas the Lab-View System calculates 19.2 degrees at $\mathrm{SNR}=4.4 \mathrm{~dB}$. On average, the relative error between these two systems is found to be within 5 degrees. It should be noted that the VC33 prototype can offer real-time estimation of DOA, whereas the Lab-View System can not.

\section{CONCLUSION}

The real-time DOA estimator VC33 is implemented based

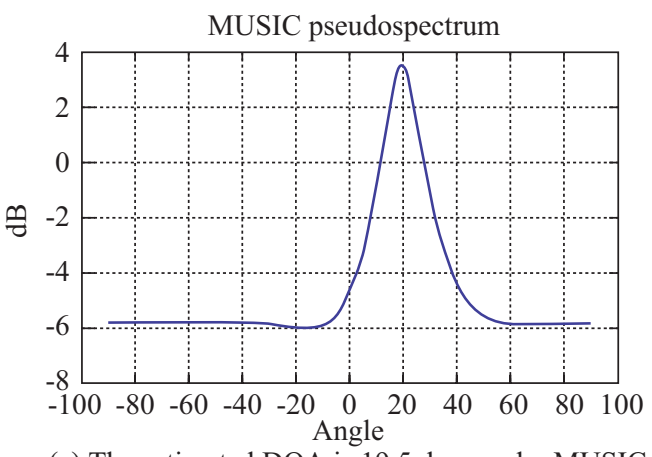

(a) The estimated DOA is 19.5 degrees by MUSIC.

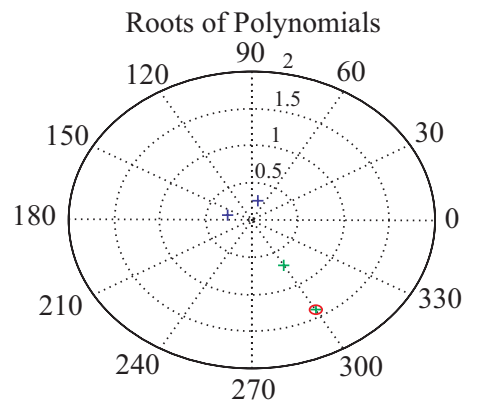

(b) The estimated DOA is 19.5 degrees by RORM.

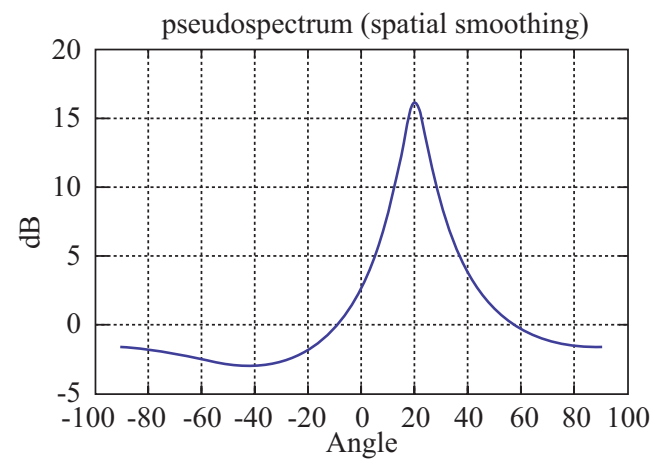

(c) The estimated DOA is 20.2 degrees by SSM.

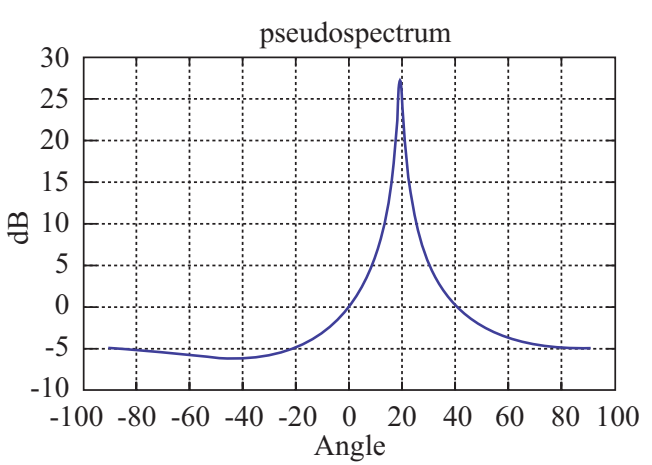

(d) The estimated DOA is 19.2 degrees by LS.

Fig. 8. The estimated DOAs at SNR $=4.4 \mathrm{~dB}$ using (a) MUSIC, (b) RORM, (c) SSM, and (d) LS.

on TMS320VC33 digital signal processor. The VC33, incorporated with a receiving hydrophone array and associated devices, can form a prototype system suitable for estimating multiple underwater acoustic sources. Sea trial experiments 


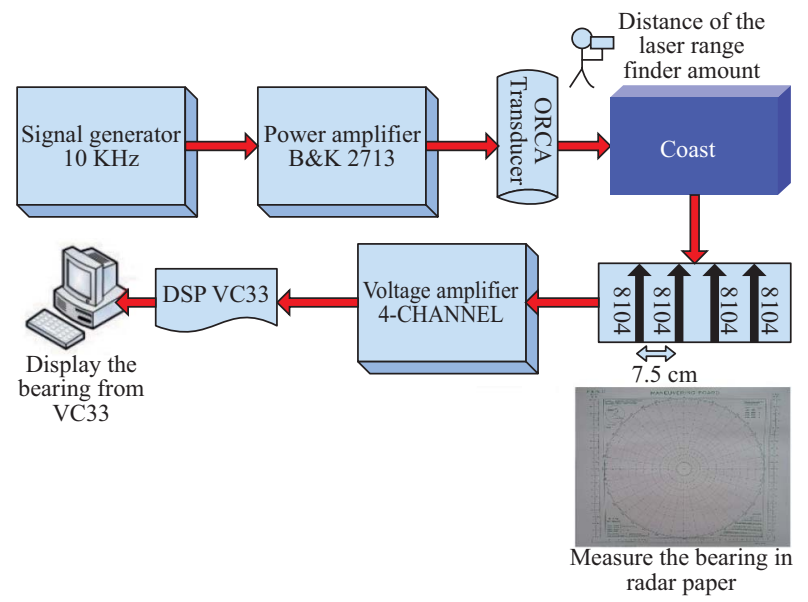

Fig. 9. Sea-trial experimental flow chart for VC33 prototype system.
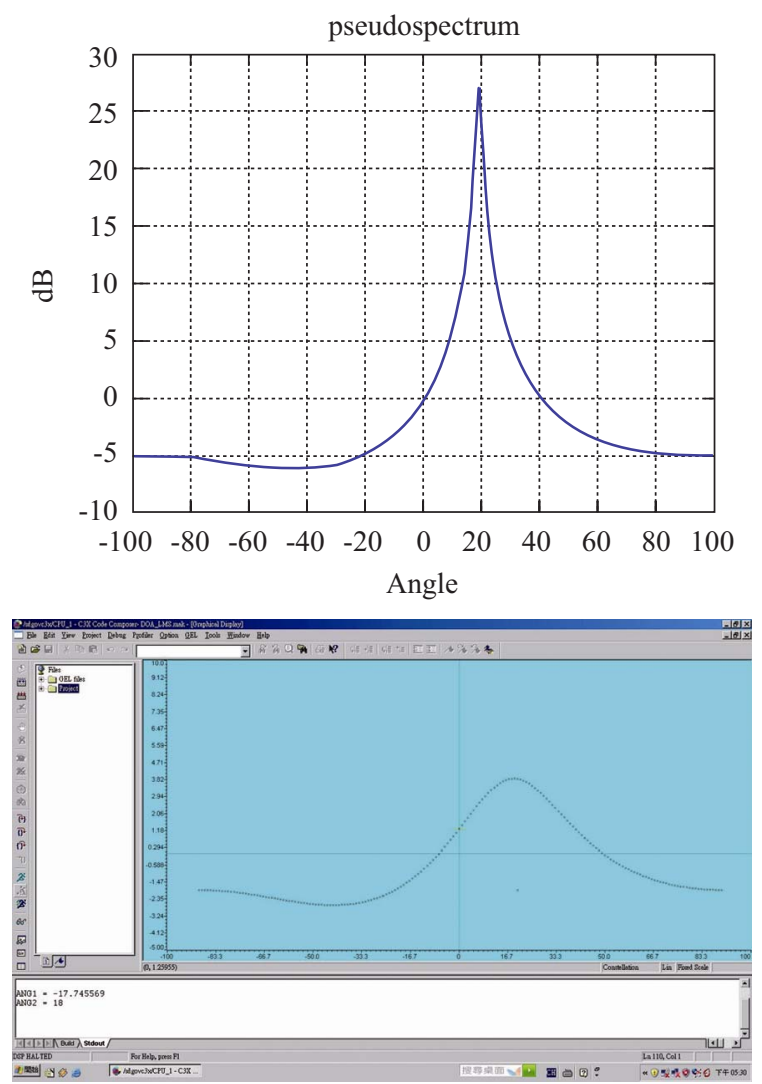

Fig. 10. Pseudo-spectrum: Lab-View (upper half), VC33 prototype (lower half). have demonstrated that the completed prototype is able to locate the directions of underwater acoustic sources in real time.

\section{ACKNOWLEDGMENTS}

This work was supported by National Science Council of TAIWAN under contracts NSC 96-2221-E-022-016- and NSC 95-2221-E-022-017-. The authors would like to thank unknown reviewers with invaluable comments and suggestions. Thanks also go to Mr. C. H. Liu, Mr. M. C. Chuang, and Mr. Y. D. Huang for their efforts in setting up the experimental equipments during the sea trial experiments.

\section{REFERENCES}

1. Barabell, A. J., "Improving the resolution performance of eigenstructure-based direction-finding algorithms," Proceeding of ICASSP, Boston, MA, pp. 336-339 (1983).

2. Chang, S.-H., Hou, S.-Y., Hung, H.-S., and Chang, S.-C., "Underwater wideband signal tracking based on predictive angle tracking algorithm," Journal of Marine Science and Technology, Vol. 13, No. 1, pp. 46-53 (2005).

3. Compton Jr., R. T. Adaptive Arrays- Concepts and Performance, Prentice-Hall, Englewood Cliffs, (1988).

4. Hung, H.-S., Hou, S.-Y., Lin, S., and Chang, S.-H., "Reduced-order Root-MUSIC for DOA Estimate," IEICE Transactions on Fundamentals of Electronics, Communications and Computer Sciences, Vol. E87-A, No. 4, pp.2160-2163 (2004).

5. Lai, W. K. and Ching, P. C., "A New approach for coherent direction -of-arrival estimation," Proceeding of the 1998 International Symposium of Circuits and Systems, Vol. 5, pp. 9-12 (1998).

6. Schmidt, R. O., "Multiple emitter location and signal parameter estimation," Proceeding of the RADC Spectrum Estimation Workshop, Rome NY, pp. 243-258 (1979).

7. Shan, T. J., Wax, M., and Kailath, T., "On spatial smoothing for direction -of-arrival estimator of coherent signals," IEEE Transaction on Acoustics, Speech, and Signal Processing, Vol. ASSP-33, No. 4, pp. 806-811 (1985).

8. TMS320VC33 Digital Signal Processor Datasheet, Texas Instruments Incorporated, (2002).

9. TMS320C3X User's Guide, Texas Instruments Incorporated (1997).

10. AD7891 Datasheet, Analog Devices Incorporated (2002).

11. LF398 Datasheet, National Semiconductor Corporation (2000).

12. IDT71V242 Datasheet, Integrated Device Technology Incorporated (2002).

13. AT29LV040A Datasheet, Atmel Incorporated (2002).

14. TMS320C $3 \mathrm{x} / \mathrm{C} 4 \mathrm{x}$ Assembly Language Tools, Texas Instruments Incorporated, Dallas, Texas (1997). 